\title{
Photonics Enabling Coherent MIMO Radar Networks
}

\author{
Filippo Scotti ${ }^{1}$, Antonio Malacarne ${ }^{2}$, Salvatore Maresca ${ }^{2}$, Paolo Ghelfi ${ }^{1}$, Giovanni Serafino ${ }^{2}$, \\ and Antonella Bogoni ${ }^{2}$ \\ ${ }^{1}$ Consorzio Nazionale Interuniversitario per le Telecomunicazioni (CNIT), via G. Moruzzi 1, Pisa, Italy \\ ${ }^{2}$ Scuola Superiore di Studi Universitari e di Perfezionamento Sant'Anna, via G. Moruzzi 1, Pisa, Italy \\ filippo.scotti@cnit.it
}

\begin{abstract}
The potential of coherent MIMO radar networks enabled by photonics is introduced. The first coherent dualband $2 \times 4$ MIMO radar experiment is presented. Range/cross-range maps demonstrate the higher cross-range resolution due to the coherence and the enhanced performance introduced by dual-band operation.
\end{abstract}

Keywords: microwave photonics, VCSEL, MIMO radar, dual-band radar.

\section{INTRODUCTION}

Since their invention, radar sensors never stopped evolving from their historical applications, such as air and naval traffic monitoring, to cover the most disparate scenarios, like on-board systems supporting aerial driving, monitoring of atmospheric conditions, deforestation and pollution factors through satellite observations, evaluation of landslide risks, just to name a few. Beside the evolution of the capabilities, and thanks to the ever increasing miniaturization and cost reduction of electronic components, radars are progressively becoming more pervasive in many of the latest high-precision applications, such as road traffic monitoring, collision avoidance systems, and control of industrial assembly lines [1]. Thus, to meet constantly higher demands imposed by those applications in terms of resolution, stability and accuracy within a coverage volume, radars have started becoming even more ubiquitous sensors [2].

Although typical radar can reach quite high range resolutions thanks to the employed large bandwidth, they present intrinsic limitations in terms of angular or cross-range resolution, which is given by the antenna. In fact in common antennas like parabolic dishes or slotted waveguide, and even a modern phased-array, the beam divergence depends on the geometric dimension of the antenna, thus there is a limit on how narrow a radio beam can be focused before the antenna size just make the system impractical. A solution to improve the angular resolution, as it happens with synthetic aperture radar (SAR) [3], consists in observing the same scene from different points of view, thus emulating a bigger antenna. Since, in many of the abovementioned scenarios, it is not feasible to continuously scan the radar position as it happens in satellite observations, an alternative relies in realizing networks of spatially distributed radars. Such a system is capable of simultaneously observing the same scene from different points of view, leading to evident benefits in the spatial accuracy of target detections.

In a recent class of radar systems, named multiple-input multiple-output (MIMO) radar, a multitude of radar sensors operate in synergy, so that signals emitted by multiple transmitters are received by multiple receivers enabling jointly elaboration of the collected data [4],[5]. By exploiting the processed information collected from different spatial positions, an excellent cross-range resolution can be achieved, which is basically independent of the characteristics of each single antenna. In addition, if a mutual phase coherence among data from each sensor is guaranteed, their centralized elaboration theoretically leads to a further improvement of detection and localization capabilities [6],[7]. However, wide distribution of sensors also causes the rise of sidelobes for each main lobe corresponding to a detected target. Some methods for optimizing the sensor distribution geometry in case of wide separation, so as to minimize the sidelobe contribution with respect to the main lobe one, i.e. sidelobe suppression ratio, are recently being proposed [8].

If spreading multiple sensors over a vast area can overcome the angular resolution limit, the range resolution suffers from spectral erosion driven by the ever-expanding wireless communication services, that leaves only sparse and narrow bands available for radar sensing. To this extent, MIMO radars transmitting multiple coherent bands can increase their bandwidth occupying the multiple narrow slices of spectrum left by the communication industry. Moreover, they may also provide additional information to enhance target classification and detection by means of improved classification algorithms [9],[10].

However, generation and manipulation of coherent RF signals at different carrier frequencies and their coherent distribution along a radar network is challenging. Photonics has proved to be extremely effective in guaranteeing frequency flexibility, with coherent multi-band signal distribution enabling coherent processing [12]. However, those solutions are based on the use of sophisticated fiber-based mode-locked lasers. Some recent works have demonstrated that direct modulation (DM) of laser diodes - in particular of vertical-cavity surface emitting lasers (VCSELs) - represents an attractive low-cost, power-efficient and robust solution for coherent distribution of analog RF signals [13]. Their high-speed direct modulation response goes well with the ever-increasing frequencies used by the new generation radars and their robustness to high temperature allows uncooled operation. In this work we demonstrate that, thanks to the coherence provided by VCSEL-based RF 

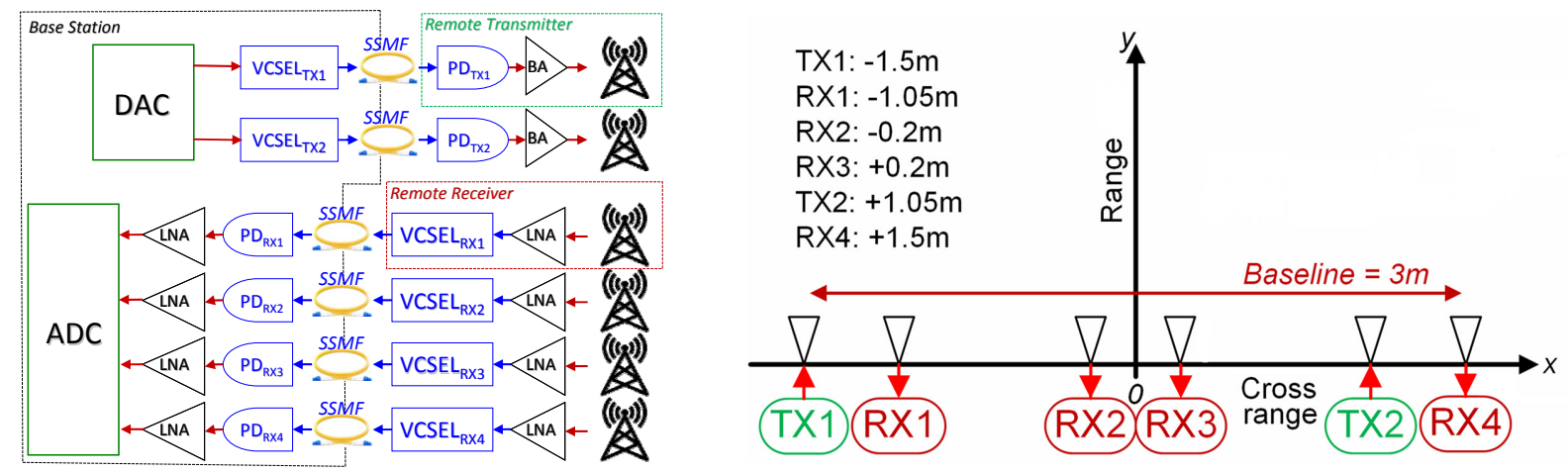

Figure 1. Experimental setup implementing the $2 \times 4$ VCSEL-based MIMO radar network, and scheme of the deployed radar sensors.

signal distribution, coherent multiband MIMO processing is successfully applied to a Radar-over-Fiber (RaoF) network, where remoting of transmitters (TXs) and receivers (RXs) with respect to a centralized base station is achieved through DM-VCSELs.

\section{VCSEL-based MIMO Radar Network Experimental Setup}

An indoor experimental setup has been realized to demonstrate the operational capabilities of a MIMO radarover-fiber system based on the hardware of the TX and RX presented in [13] and is reported in Fig. 1. A base station (BS) delivers the RF signals to two remote TXs and collects the RF echo signals from four remote RXs, connected by standard single mode fibers (SSMF), such that a $2 \times 4$ MIMO system is realized.

In details, two channels of a Fujitsu digital-to-analog converter (DAC) with a sampling rate of $60 \mathrm{GS} / \mathrm{s}$ directly drive two $1.3 \mu \mathrm{m}$ VCSELs transferring the radar waveforms to the optical domain. Once these signals reach the transmitters, they are converted back to the electrical domain via $10 \mathrm{GHz}$ photodiodes, amplified up to $20 \mathrm{dBm}$ by means of booster RF amplifiers (BA), and transmitted through commercial antennas with a gain of $12 \mathrm{dBi}$ in the frequency range 6-11 GHz. On the other hand, the echoes of the signals are collected by four more receiving antennas that feed $40 \mathrm{~dB}$-gain RF low-noise electrical amplifiers (LNA) that properly enhance the weak received signal to directly modulate four more VCSELs. Once at the base station, the received echoes returns to the electrical domain and, after being amplified with proper LNAs, they are digitized by the four channels of a $40 \mathrm{GS} / \mathrm{s} 13 \mathrm{GHz}$ analog bandwidth real time oscilloscope (Teledyne LeCroy SDA-813Zi-A).

In the proposed dual-band experiment, $\mathrm{TX}_{1}$ and $\mathrm{TX}_{2}$ transmit repetitive $273 \mathrm{~ns}$-long linearly $400 \mathrm{MHz}-$ bandwidth frequency-modulated continuous-wave (LFM-CW) signals with carrier frequencies of $8 \mathrm{GHz}$ and $9 \mathrm{GHz}$. The signals are contemporaneously transmitted by $\mathrm{TX}_{1}$ and $\mathrm{TX}_{2}$, but they are separated in the frequency domain to limit mutual interferences and up- and down-sweep LFM-CW ensure an appropriate signal orthogonality. Specifically, when $\mathrm{TX}_{1}$ transmits at $8 \mathrm{GHz} \mathrm{TX}_{2}$ transmits at $9 \mathrm{GHz}$. At the successive waveform repetition interval, $\mathrm{TX}_{1}$ transmits at $9 \mathrm{GHz}$, instead, while $\mathrm{TX}_{2}$ transmits at $8 \mathrm{GHz}$. Then, the transmission scheduling restarts following this periodicity.

A third DAC channel was used to trigger the ADC (oscilloscope) and both DAC and ADC were referenced with a common $10 \mathrm{MHz}$ tone, so that coherence is assured.

As also reported in Fig. 1, the antennas are placed on a 1D 3m-long baseline, thus enabling 2D imaging (x-y plane). The exact location of each antenna is included in the figure. As a target, a cylinder can with a radar cross section (RCS) of $-7 \mathrm{dBsm}$ (assumed omnidirectional on the range/cross-range plane) at a distance of $3.6 \mathrm{~m}$, was used.

The acquired signals are processed as follows. First, the four received signals are cross-correlated with the two reference waveforms (i.e., the ones employed at the two TXs), thus obtaining eight vectors, one for each TX/RX virtual channel. It is worth noticing that during this operation the system delay of each TX/RX virtual channel is compensated for different delays caused by cables and circuitry. In addition, the amplitudes of the received signals are compensated in post-processing.

At this point, since the complex cross-correlation vectors are functions of the TX-target-RX travelled time, which is in turn proportional to the bistatic range, they can be mapped via geometric transformation into complex bi-dimensional (2D) matrices. In fact, given the position of the generic TX and RX element, which act as the two foci, the peaks displayed within the cross-correlation vector transform into eight ellipsoidal wave fronts by varying the range and the cross-range, as depicted in Fig. 2.

Finally, depending on the required output, non-coherent and coherent MIMO processing can be performed. In the first output type, the square modules of the eights complex channel matrices are summed (i.e., noncoherent summation). In the second output type, the each of complex matrices is first multiplied by a further "phase-alignment" matrix, which compensates for the phase shifts due to the different signal travel delays in free 

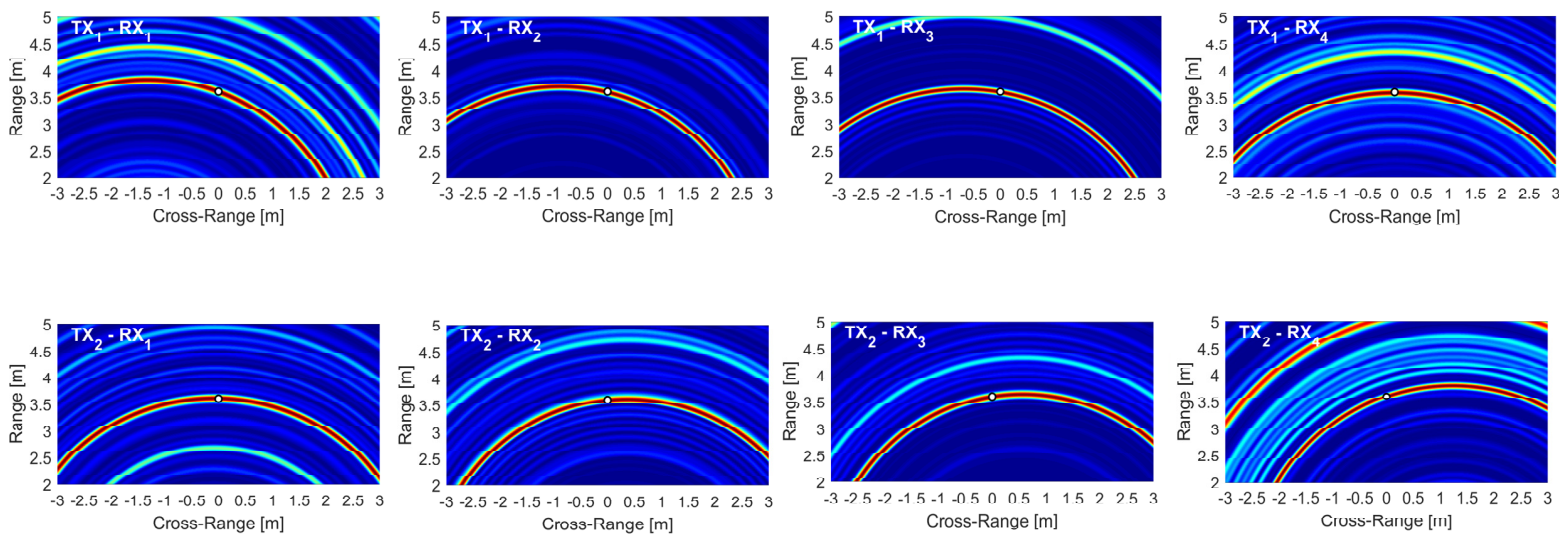

Figure 2. Color-coded normalized squared modulus of the cross-correlation vector calculated at each bistatic radar channel after 2D mapping.

space. Finally, the resulting phase-aligned matrices are summed together, giving a single matrix representing the observed scenario. The MIMO radar processing tools have been developed through Matlab codes running offline, after data acquisition.

\section{Dual-band VCSEL-based MIMO Radar Network Experiment}

To assess the 2D imaging capability of the proposed photonics-based radar network, as a first step the system has been configured to perform single-band non-coherent MIMO processing (whose result is almost identical to the non-coherent dual-band case, shown in Fig. 3A). The single radar detections of Fig. 2 do not provide any angular information, since target likelihood functions are spread over bistatic iso-range ellipsoids, but the superposition of the eight maps, i.e. the non-coherent MIMO output, provide an angular resolution of about $2 \mathrm{~m}$, depending on the geometry of the radar array with respect to the target position.

With the coherent MIMO processing on a single carrier frequency, each of the 2D map is phase-aligned, and the result, shown in Fig. 3B, presents a strong improvement in cross-range resolution with respect to the noncoherent approach. As a matter of fact, while in a single-band radar system the range resolution, which is determined by the signal bandwidth, remains almost unchanged, the angular resolution can be calculated as the ratio between the wavelength of the RF carrier and the baseline length. In this case, being the wavelength about $3.5 \mathrm{~cm}$ and the baseline $3 \mathrm{~m}$, the theoretical cross-range resolution at $3.6 \mathrm{~m}$ range is about $2.6 \mathrm{~cm}$, in excellent agreement with the results.

However, many large sidelobes can also be spotted around the main peak. The high and numerous sidelobes are due to the limited MIMO array configuration, the geometry of the array with respect to the target, and the small fractional bandwidth. Anyway, their relative position depends on the wavelength of the radar signal, thus multi-band processing can be helpful.

The improvement in case of dual-band operation with respect to single-band operation is shown in Fig. 3C. Here, the target-originated peak can be clearly observed, while the secondary undesired replicas are almost vanished. The achieved experimental results demonstrate the importance of radar signal coherence for increasing the cross-range resolution and preventing the raising of additional sidelobes due to possible channel amplitude/delay mismatches. In fact, when the necessary bandwidth cannot be fully exploited, the multi-band operation offers more degrees of freedom for reducing the sidelobes that origin from the sparse configuration of the sensors.

(A)

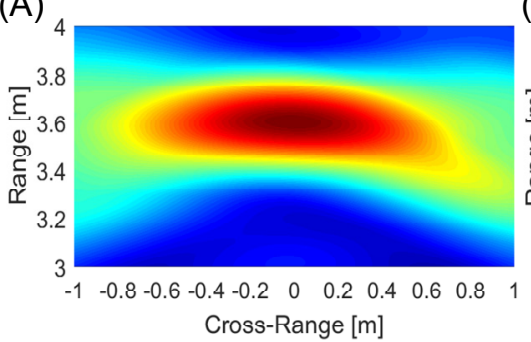

(B)

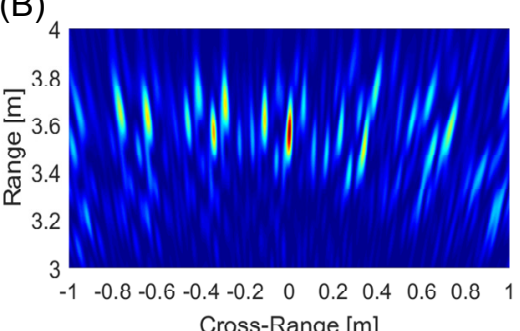

(C)

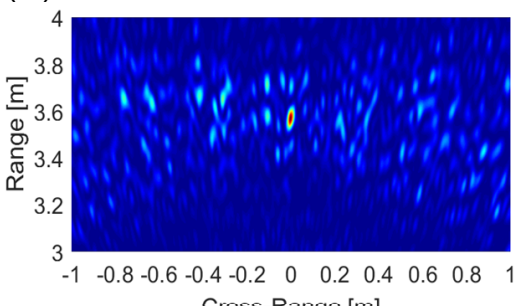

Figure 3. Color-coded normalized MIMO ambiguity function in the range/cross-range Cartesian space for: (A) on-coherent MIMO processing of dual-band real data; (B) coherent MIMO processing of real data for the single-band configuration; (C) coherent MIMO processing of dual-band real data. 


\section{CONCLUSIONS}

The potential of a multi-band coherent radar network has been experimentally demonstrated. Distribution of the $\mathrm{RF}$ signals from and to a unique base station guarantees phase coherence, allowing the so called coherent multiple-input multiple-output (MIMO) processing. The use of directly modulated VCSELs allows an easy and cheap signal distribution, even over multiple frequency bands, without affecting the performance. A coherent dual-band operation with two $400 \mathrm{MHz}$-wide bands $1 \mathrm{GHz}$ apart demonstrated a cross-range resolution down to $2.7 \mathrm{~cm}$. Authors believe the presented results clearly state that the proposed VCSEL-based signal distribution represents an effective way to ensure phase stability and coherence among signals - also at multiple frequency bands - in a centralized radar network, therefore enabling coherent multi-band MIMO processing. The proposed VCSEL-based RF signal distribution through optical links prevents electromagnetic interference issues, reduces the encumbrance of each link and is a low-cost and power efficient solution perfectly fitting on-board automotive scenarios. In addition, the use of VCSELs with emission frequency in the $1.3 \mu \mathrm{m}$ - regime avoids significant signal distortion induced by chromatic dispersion of standard fiber, enabling, in principle, TX/RX remoting up to kilometer reach, consequently making the presented solution attractive for other application areas too.

\section{ACKNOWLEDGEMENTS}

This work has been partially supported by Veoneer. Authors would like to thank Olof Eriksson from Veoneer for technical support and Christian Neumeyr from Vertilas GmbH for having provided the TO-can VCSELs.

\section{REFERENCES}

[1] M. S. Greco, J. Li, T. Long, and A. Zoubir, "Advances in radar systems for modern civilian and commercial applications: Part 1 [From the Guest Editors],” IEEE Signal Processing Magazine, vol. 36, no. 4, pp. 13-15, Jul. 2019

[2] J. J. Alter, R. M. White, F. F. Kretschmer, I. D. Olin, and C. L. Temes, "Ubiquitous radar: An implementation concept," in Proc. 2004 IEEE Radar Conf., Philadelphia, PA, USA, 2004, pp. 65-70.

[3] Moreira, P. Prats-Iraola, M. Younis, G. Krieger, I. Hajnsek, and K. P. Papathanassiou, "A tutorial on synthetic aperture radar," IEEE Geoscience and Remote Sensing Magazine, vol. 1, no. 1, pp. 6-43, Mar. 2013.

[4] H. Godrich, A. M. Haimovich, and R. S. Blum, "Target localization accuracy gain in MIMO radar-based systems," IEEE Transactions on Information Theory, vol. 56, no. 6, pp. 2783-2803, Jun. 2010.

[5] E. Fishler, A. Haimovich, R. Blum, D. Chizhik, L. Cimini, and R. Valenzuela, "MIMO radar: An idea whose time has come," in Proc. 2004 IEEE Radar Conference, Philadelphia, PA, USA, 2004, pp. 71-78.

[6] A. M. Haimovich, R. S. Blum, and L. J. Cimini, "MIMO Radar with Widely Separated Antennas," IEEE Signal Processing Magazine, vol. 25, no. 1, pp. 116-129, 2008.

[7] N. H. Lehmann, A. M. Haimovich, R. S. Blum, and L. Cimini, "High resolution capabilities of MIMO radar," in Proc. 2006 Fortieth Asilomar Conference on Signals, Systems and Computers, Pacific Grove, CA, 2006, pp. 25-30.

[8] L. Lembo, P. Ghelfi, and A. Bogoni, "Antenna position optimization in a MIMO distributed radar network through genetic algorithms," in Proc. 20th International Radar Symposium (IRS), Ulm, Germany, 2019, pp. 1-6.

[9] S. Maresca, D. R. Sanchez-Jacome, A. Bogoni, and P. Ghelfi, "Information diversity in coherent MIMO radars," in Proc. 17th European Radar Conference (EuRAD 2020), Utrecht, Netherlands, 16-18 Sep. 2020.

[10] P. Van Dorp, R. Ebeling, and A. G. Huizing, "High resolution radar imaging using coherent multiband processing techniques," Proc. IEEE Radar Conf., Washington, DC, USA, 2010, pp. 981-986.

[11] F. Scotti, F. Laghezza, P. Ghelfi and A. Bogoni, "Multi-band software-defined coherent radar based on a single photonic transceiver," IEEE Transactions on Microwave Theory and Techniques, vol. 63, no. 2, pp. 546-552, Feb. 2015.

[12] P. Ghelfi, F. Laghezza, F. Scotti, D. Onori, and A. Bogoni, "Photonics for radars operating on multiple coherent bands," Journal of Lightwave Technology, vol. 34, no. 2, pp. 500-507, 2016.

[13] A. Malacarne et al., "A ultrawide-band VCSEL-based radar-over-fiber system," in Proc. 2019 International Topical Meeting on Microwave Photonics (MWP), Ottawa, ON, Canada, 2019, pp. 1-4. 